\title{
EXPERT REVIEWS \\ Neuromuscular stimulation after stroke: from technology to clinical deployment
}

Expert Rev. Neurother. 9(4), xxx-xxx (2009)

Maarten J IJzerman ${ }^{\dagger}$ Gerbert J Renzenbrink and Alexander $\mathrm{CH}$

\section{Geurts}

${ }^{\dagger}$ Author for correspondence

University of Twente, School for Management \& Governance,

Department of Health

Technology \& Services Research, PO Box 217, 7500 AE Enschede,

The Netherlands

Tel.: +31534893684

m.j.ijzerman@utwente.nl

Since the early 1960s, electrical or neuromuscular electrical stimulation (NMES) has been used to support rehabilitation of stroke patients. One of the earliest applications of NMES included the use of external muscle stimulation to correct drop-foot after stroke. During the last few decades various clinical applications have been used for the upper and lower limb. Despite a growing body of literature on the use of NMES, its application in stroke is still limited to a few clinical groups that provide dedicated clinical services. Some explanations for the limited use are the sometimes conflicting clinical evidence, the size of the effects or the complicated use of the technology itself. This review points out three directions for future research. First, we need to expand our knowledge on brain plasticity and the use of different electrical stimulation strategies to modulate the neural system. Second, we foresee an increase in therapies combining different training principles, for example, the combination of NMES and robotics or neuromodulating drugs. Finally, with the ever-increasing pressure on healthcare budgets, it is expected that clinical and economic evidence will become more relevant in transferring these interventions to a wider community.

KeYwords: drop-foot implant $\bullet$ medical technology assessment $\bullet$ motor relearning $\bullet$ neural prostheses $\bullet$ neuromodulation • neuromuscular electrical stimulation • outcomes research $\bullet$ pain $\bullet$ stroke

\section{Neuromuscular electrical stimulation in stroke}

It is clear that Luigi Galvani served as the founder of modern neurophysiology and the use of electrical current to control paralyzed muscles [1]. In particular, the discovery of the ability of electric current to stimulate paralyzed muscles of a frog is often referred to as the starting point of neuromuscular electrical stimulation (NMES; see Box 1) in stroke research. The first applications of NMES in clinical medicine were reported in the early 1960s. After extensive laboratory and animal testing, the first pacemaker was implanted in 1958.

The first paper reporting on the use of NMES in neurological patients was published in 1961. Liberson described the use of electrical current to stimulate the dorsal flexors of the foot in stroke patients to prevent drop-foot during the swing phase of gait [2]. The technique reported by Liberson can be regarded as a 'neural prosthesis', that is, the stimulation is used to generate muscular contractions and, hence, to substitute for lost functional movements. Although some colleagues prefer the word 'functional electrical stimulation' (see Box 1) for this type of neurostimulation, the term 'neural prostheses' better describes the category of medical devices using electrical current to compensate for a deficient neural system.

Several reviews and studies have been published on the use of NMES in different neurological disorders [3-10]. In stroke rehabilitation, NMES can be used twofold; besides being a neural prosthesis, for example to prevent dropfoot, electrical current can be used to modulate neural activity to either regain voluntary muscle contractions or to prevent abnormal muscle reactions, for example due to spasticity. Somewhat complicating, however, is the possibility that both goals can be pursued simultaneously and that the last few decades showed that such an interaction has been achieved [11]. Indeed, a peroneal nerve stimulation system may not only stimulate muscles to prevent drop-foot, it may also modulate the neuromuscular system in the long term, and thus yield a 'carryover effect'. Such carryover or therapeutic effects of neurostimulation result from intensive training with a neural prosthesis and typically last until after 


\section{Box 1. Glossary of terms.}

Neuromuscular stimulation: stimulation of muscles through electrical current.

Functional electrical stimulation: neuromuscular stimulation used for functional purposes, sometimes used interchangeably with neuromuscular stimulation.

Neural prosthesis: an artificial system bypassing the neural system to restore lost body functions by providing functional movement patterns using electrical stimulation.

Neuromodulation: changing normal and abnormal neural activity in both sensory and motor nerves to manipulate function. Surface stimulation: neuromuscular stimulation applied through the skin

Percutaneous stimulation: electrodes passing the skin to be implanted in either motor points or in the proximity of muscles.

neural prosthesis use has stopped. On the other hand, some forms of NMES, such as of the upper limb muscles, have primarily been developed for their carryover effects and can best be referred to as therapeutic modalities.

It is this complexity of momentary and prolonged effects that hinders a clear interpretation of the clinical efficacy of most neurostimulation systems and the disentanglement of the specific effects of NMES in terms of either therapeutic or prosthetic components. In addition, actual clinical use of NMES and neural prostheses appears to be limited, and only few clinical experts acknowledge the benefits [12]. Moreover, this may have been a result of the field emerging mainly from biomedical engineering, which did not sufficiently take into account existing theories in motor relearning [11], nor the current requirements of evidence-based medicine [13]. Today, several reviews and clinical practice guidelines on stroke management are published [14-17]. However, these guidelines are not always helpful in applying NMES. For instance, the American Heart Association (AHA)/American Stroke Association (ASA) guidelines [17] and the Veterans' Affairs (VA)/Department of Defense (DoD) recommendations [16] were based on only a few clinical papers, of which one was another review. In other cases, there are conflicting recommendations between either review papers or guidelines. For instance, the Evidence Based Review of Stroke Rehabilitation (EBRSR) guidelines do not support the use of electrical stimulation for the hemiplegic arm [201], whereas others do support that particular application [14].

In the present review we therefore wish to provide a critical assessment on the use of both neural prostheses and NMES in stroke rehabilitation. Mainly, three applications will be described: peroneal neurostimulation for foot-drop, NMES of paretic upper limb muscles and neuromodulation of severe shoulder pain. Each of these three applications will be reviewed in terms of technological developments, clinical evidence and problems related to the deployment of these techniques. The last section of the review will introduce the concept of neuroelectronic interfacing in stroke rehabilitation. This concept introduces the much wider field of neuromodulation and neuroaugmentation in sensitizing brain tissue to receive motor relearning input.

\section{Neural prostheses for drop-foot}

\section{Technology \& available devices}

A drop-foot is a common complication after stroke, preventing a safe and stable walking pattern. Drop-foot can be defined as a significant weakness or absent activity of ankle and toe dorsiflexors. These muscles assist in clearing the foot during the swing phase and control plantar flexion of the foot on heel strike. Drop-foot following stroke is thought to be caused mainly by poor active control of the anterior tibial muscle combined with increased and inappropriate tone in the dorsal muscles of the leg, particularly the calf $[18]$.

The basic idea of a drop-foot stimulator is to activate the ankle dorsal flexors and the evertors of the foot (i.e., the anterior tibial and peroneal muscles) by stimulating the deep and superficial branches of the common peroneal nerve. Control over the timing of the stimulation is usually obtained through a heel switch placed under the heel within the shoe, although other control signals, such as accelerometers, can also be used $[19,20]$. Clearly, surface stimulation has limited selectivity and delivers a pulse train to both branches of the peroneal nerve. Other disadvantages of surface stimulation include problems with electrode placement, pain, skin irritation and the time required to put the device in place.

Since 1961, both surface and implantable systems have been introduced to manage drop-foot [21]. More recently, several new neural prostheses have been commercially introduced for dropfoot. Hanger Orthopaedic group introduced the WalkAid ${ }^{\circledR}$ in early 2006, a surface peroneal nerve stimulator controlled by a tilt sensor attached just below the knee [202]. No clinical reports have been published about the device, however, Weber $e t$ al. reported on the use of BION ${ }^{\mathrm{TM}}$ as an implantable stimulator to be used with a WalkAide stimulator [22].

The NESS L300 ${ }^{\mathrm{TM}}$ is a new generation surface electrical stimulation systems for drop-foot [203]. It is controlled by a heel switch that wirelessly communicates with the stimulator that is integrated within a semi-rigid orthosis positioned just below the knee. Two implantable stimulators have become available recently. The ACTIGAIT ${ }^{\mathrm{TM}}$ system [204] was developed in Denmark and is distributed by Otto Bock, Ltd [20]. The second implantable system, STIMUSTEP ${ }^{\mathrm{TM}}$, was developed by the Twente group and is being distributed by Finetech Medical Implants, Ltd [205]. So far, approximately 45 STIMuSTEP units have been produced and tested in both Phase II and Phase III clinical trials. The ACTIGAIT system uses a four channel cuff electrode around the common peroneal nerve, whereas the STIMUSTEP ${ }^{\mathrm{TM}}$ uses two epimysial electrodes that are inserted into the nerve sheets of the deep and superficial branches of the common peroneal nerve.

\section{Clinical evidence}

Since the early 1990s, the Odstock Dropped Foot Stimulator has been probably one of the most widely used and best documented surface stimulation systems [23]. Several studies from their group have shown an increased walking speed and a decreased energy expenditure measured with the physiological cost index 
[24]. In addition, the perception of the users was that the Odstock Dropped Foot Stimulator was of clinical benefit and, therefore, the compliance was very high [25].

In a systematic review, the results of eight studies were analyzed to asses the neural prosthetic effect of peroneal stimulation on walking in stroke survivors with a drop foot [26]. The pooled effect size for increase in walking speed was $0.13 \mathrm{~m} / \mathrm{s}(95 \% \mathrm{CI}$ : $0.07-0.2 \mathrm{~m} / \mathrm{s}$ ) or $38 \%$ (95\% CI: $22.18-53.8 \%$ ). Another metaanalysis was published where the therapeutic effect of peroneal stimulation on walking speed in stroke survivors was determined [27]. A significant mean improvement of $0.18 \mathrm{~m} / \mathrm{s}$ was found, indicating clear effectiveness of NMES on walking speed after stroke.

Only recently, a clinical study has been reported on the L300 neural prosthesis [28]. They found an average improvement in walking speed of approximately $30 \%$ compared with no treatment, using a within-subjects design. Also recently, new clinical studies on the implantable ACTIGAIT and STIMuSTEP ${ }^{\mathrm{TM}}$ systems have been published [29-31]. Burridge et al. reported a Phase II clinical trial on the ACTIGAIT stimulator. They performed an uncontrolled trial in 13 patients and concluded that ACTIGAIT is safe [29]. Kottink et al. evaluated the STIMuSTEP in a Phase III randomized controlled trial (RCT) in 29 stroke survivors (ankle-foot orthosis [AFO] vs implant) on both the orthotic and the carryover effects. They reported a significantly increased walking speed of $20 \%(\mathrm{p}<0.01)$ in the implant group [31]. Further analysis of their data did not show a clear therapeutic effect, although electromyographic recording showed an increased muscular output after 26 weeks of stimulation [30].

\section{Implementation barriers}

It has taken nearly 50 years to find clinical evidence on the effect of drop-foot stimulation in stroke. Moreover, compared with cardiac pacing, the implementation of drop-foot stimulation has appeared more difficult. Different reasons could be responsible for this delay, including technological difficulty related to timing and control of the stimulation, the debate about the possible clinical outcomes, crosscultural differences and patient preferences. In the past few decades, many of the developments had a clear technological drive, such as new electrodes, encapsulation of implants, feedback systems and others. The clinical need was less clear as AFOs were quite well accepted as the primary choice of support in stroke [32,33]. In addition, clinical studies have used many secondary outcome measures to address the beneficial effects. The reviews by Kottink et al. and Robbins et al., as well as the recently published clinical trials, emphasize walking speed as the primary end point, because functional outcome measures were less consistently used in clinical reports.

Sheffler $e t a l$. compared AFO and drop-foot stimulation and concluded that functional ambulation may not be that different [34]. Besides walking speed and functional ambulation, one may have an interest in overall health-related quality of life and preferences of users toward drop-foot stimulation. So far, only one study has measured health-related quality of life using the Short Form 36 (SF36), but the lack of a control group hinders a clear interpretation of the conclusions [35]. Also, one may argue whether or not the SF36 will be responsive in detecting differences in health-related quality of life [36].

Although level I evidence exists for a higher walking speed after using drop-foot stimulation, clear clinical practice guidelines cannot be developed from these findings. In 2004, the International Society for Prosthetics and Orthotics (ISPO) organized a consensus meeting on orthotic management of the foot after stroke [37]. The ISPO meeting agreed on the beneficial effect of NMES on walking speed and physiological cost index (level II evidence; i.e., RCTs), but concluded that the ultimate decision for either AFO or stimulation depends on the patient. Importantly, the National Institute for Clinical Excellence (NICE) has considered the option of electrical stimulation for drop-foot of neurological origin. The Interventional Procedure Application Committee (IPAC) has produced a consultation document [206]. Based on this consultation document, NICE will deliver clinical guidance and advise the NHS regarding the use of drop-foot stimulation.

In an attempt to understand the requirements of these technologies and to put drop-foot stimulation in a wider context, van Til et al. have been trying do identify key characteristics that influence medical decision making [38]. By performing a series of experiments, they have been able to select key attributes relevant to decision making by professionals and compared five therapeutic approaches (surface and implanted drop-foot stimulation, orthopedic shoes, tendon surgery and AFO) to treating drop-foot. They quantified responses of an expert panel using analytic hierarchy process, a multiple-criterion decision analysis technique. They concluded that the most preferred treatment by experts was the split-tendon tibial tendon transfer, followed by an AFO, orthopedic shoes, surface and implanted stimulation [38]. It must be concluded, however, that this surgical procedure is only suitable in well-selected patients with predominant varus deformity in swing phase and sufficient residual strength of the anterior tibial muscle.

\section{NMES for training of impaired upper limb muscles Technology \& available devices}

A second large field of NMES in stroke concerns the function of the upper limb. Compared to drop-foot stimulation, the application of electrical stimulation as a neural prosthesis has not yet led to clinical success. Chae and Hart have tried percutaneous stimulation of the upper limb for grasp and opening of the hand [39]. The main conclusion at present is that the impaired motor system and the complexity of pathological motor control of the upper limb prevents appropriate application of neural prostheses. It may be possible to develop a neural prosthesis for the upper limb, but it is necessary to control the pathological neural activity [40].

A large body of work has been carried out on NMES as a therapeutic application aiming at motor relearning. In general, training with NMES is expected to result in increased voluntary activity by plasticity of the neural system. Except for these neuroplastic effects, many other benefits have been reported, including 
spasticity reduction, increased blood flow and reduced edema [41,42]. These effects may be related to the electrically induced muscle activity and subsequent reflex modulation.

The use of NMES in motor relearning has been described extensively in various uncontrolled trials, RCTs and systematic reviews. Usually, NMES is applied through electrodes at the wrist extensors and the stimulation intensity is just above the motor threshold. There are, however, other approaches, such as the mesh glove using subthreshold stimulation [43] and transcutaneous electrical nerve stimulation (TENS) for pure sensory stimulation.

The main body of work has been performed on NMES, provoking muscle contraction. NMES can be applied through a preprogramed sequence (i.e., cyclic stimulation) or using other means of initiation (i.e., electromyogram [EMG]-triggered).

It needs to be emphasized that NMES of the upper limb is 'just another tool' that needs to be evaluated like any other neurorehabilitation approach. It might be assumed that neural plasticity effects will cause equivalent effects on clinical scales. However, to date, several unsolved issues remain and, although functional MRI (fMRI) changes have been seen after using neuromuscular stimulation (NMS), it is still not clear whether there is a causal relation between NMS and improved motor excitability [44-47].

\section{The clinical evidence for motor relearning effects using NMES}

Several review papers have been written about the expected benefit of NMES in hemiparesis [3,5,7,40,48-60]. All reviews evaluated at least one particular application of NMES. Some of them compared the effects with various other therapeutic approaches or provided a general overview of NMES and neural prostheses in either stroke or spinal cord injury.

However, three different RCTs on TENS for the upper extremity reported conflicting evidence of beneficial effects as assessed by the Barthel index and motor control [61-63]. With respect to NMES, De Kroon et al. performed a systematic review and included six RCTs comparing NMES of the upper extremity. They performed a methodological and qualitative comparison of the included papers and did not pool the data owing to heterogeneity. They concluded that some evidence exists for improved motor control. However, functional gain was not very likely [50]. Since the review by de Kroon, a few more RCTs have been published and at least the same amount of reviews [64-68]. A recent Cochrane review divided the clinical studies into four categories, those evaluating NMS versus 'doing nothing', NMS versus placebo, NMS versus 'usual therapy' and NMS versus another application of NMS [69]. The authors showed large effect sizes, particularly for studies comparing NMS with no treatment, yet they concluded that there was insufficient evidence to make clinical recommendations for using NMES in particular subgroups of patients and for specific therapeutic approaches.

One important problem is that noone demonstrated the specific and intended (carryover) effects of NMES. The different strategies all evoke repetitive movements, which is probably the most important therapeutic determinant. In addition, many study outcomes were confounded by other, nonspecific influences, such as differences in treatment intensity between the experimental and control groups. Moreover, not all reviews were consistent concerning the effect of NMES in stroke rehabilitation of the upper extremity.

The main conclusion from these studies is that there seems to be some therapeutic effect of NMES on upper limb function after stroke, especially in patients with some residual extensor activity of the wrist and fingers, but that the quality of the clinical research is generally poor and that evidence is based on many different (secondary) outcome measures.

\section{In search of specific effects: functional- \& EMG-triggered stimulation}

In the past few decades several new approaches have been developed and evaluated in small trials. One general conclusion is that any rehabilitation intervention needs to be task-oriented, repetitive, of sufficient intensity and providing feedback [70-72].

We may, therefore, conclude that the passive repetitive stimulation paradigms used in many NMES studies do not really meet the aforementioned requirements, especially not those concerning task-specific orientation and the need for feedback. In this regard, we need to distinguish between the severely impaired and those having some volitional control of the upper limb. Therefore, for the less affected patients with residual motor control, different attempts have been made to combine NMES with other, more intensive, training approaches. For instance, combining NMES with functional exercise [73], robotics [74] or the use of NMES in home exercise programs [75]. An example of a patient friendly integration of NMES with a hand-wrist orthosis is the NESS H200 ${ }^{\mathrm{TM}}$. This device can produce cyclic stimulation of forearm flexors and extensors, but preprogrammed movement patterns can also be used to enhance functional goal-oriented training [76]. Moreover, the user-friendly design facilitates therapy compliance, treatment intensity and long-term use [42].

Some RCTs investigated specific aspects of NMES to find evidence for differential effects. For instance, De Kroon compared stimulation of the wrist extensors with stimulation of the wrist flexors and did not find a difference on the action research arm test [67]. Besides, De Kroon et al. reviewed the various stimulation settings used in different applications of electrical current [52]. They found indications that studies using EMG-controlled stimulation had better treatment outcomes. The results should be interpreted with caution as the review did not take into account all variation in study population, types and intensity of treatment, and outcome assessment.

One other proposed method to increase therapeutic outcomes is to combine the stimulation with voluntary muscle activity recordings. The idea of EMG-controlled stimulation is that a patient is actively involved in the training and that stimulation does not start before a patient reaches a preprogramed level of voluntary contraction. Several reviews and clinical studies have been conducted comparing EMG-triggered stimulation to 
either conventional stimulation or other therapeutic approaches. The results of these studies are, again, controversial. One RCT compared EMG-triggered with cyclic NMS and concluded that there was no difference on functional outcomes [66]. Rather than just using a passive stimulation strategy, it is probably better to apply the stimulation in a more intense training combining various therapeutic paradigms. A recent trial investigated the effect of movement imagery combined with EMG-triggered feedback compared with a conventional NMES program [65]. It concluded that there was no difference between the two therapeutic approaches. Another approach was introduced by Cauraugh and Kim, who coupled two protocols during training $[77,78]$. The coupling refers to bimanual exercises in which the intact limb assists the impaired limb during training combined with EMG-triggered NMS. The theoretical advantage would be that bilateral training exercises stimulate symmetrical activation of the cerebral hemispheres.

In addition, two reviews on EMG-triggered NMES have been published. Bolton et al evaluated EMG-triggered stimulation in six different studies of 47 subjects in the intervention group and 39 in the control group. They calculated a positive mean standardized effect size of 0.82 [64]. Meilink et al. reviewed EMGtriggered NMS compared with usual care and concluded that one was not superior to the other [68]. They included 157 patients in total. The difference in their conclusion can be explained by the different studies reviewed and the inclusion of noncontrolled studies by Bolton et al [68].

Similar to Cauraugh, other approaches have been proposed to involve the unaffected upper limb in the training of the affected upper limb. Knutson et al. developed the contralaterally controlled functional electrical stimulation training, a technique where they used the opposite wrist angle as a signal to start the stimulation. By doing so, they aim at combining the effects of NMES, bimanual symmetric exercises, and active and goal oriented training [79]. Their first experiences with this technique in a few stroke patients justify further clinical investigation.

It is not very surprising that most studies investigating NMES after stroke have used surface stimulation. The therapeutic and temporary nature of the stimulation does not really provide arguments for implanted stimulation. However, a decade ago the BIONTM was introduced as a simple device delivering electrical current locally at the place of insertion [207]. The BION can be injected under local anaesthesia. It has been shown that a BION microstimulator can be implanted safely $[80,81]$. The first Phase I clinical study in seven subjects demonstrated that upper limb functions can indeed be trained and that the application is feasible. It remains to be determined what the future role of implanted stimulation systems will be in the rehabilitation of the upper limb after stroke.

\section{Implementation barriers}

In general, NMES has been used quite a lot in physiotherapy because it is relatively cheap and easy to apply. However, although NMES for motor relearning after stroke has been studied frequently, its use has not been spread widely. One of the causes may be that the available evidence is still limited and that specific effects cannot be distinguished from aspecific effects. RCTs and systematic reviews provide both positive and negative outcomes. One of the reasons for this controversy is the heterogeneity, of the study population, intervention and outcome measures used. Even systematic reviews and meta-analyses use different approaches in how to deal with this heterogeneity. A typical problem is the relatively small sample sizes (typically ranging from six to 30 patients per group) and the related risk of type II errors (false-negative outcomes).

Most studies reported on NMS in chronic stroke survivors. If neural plasticity effects are to be expected, one would be more interested in starting earlier after stroke. Moreover, different studies reported that the initial degree of motor paralysis seems to be the best predictor of recovery [82].

Given these remarks, what would be the future role of NMES of the upper extremity in stroke rehabilitation? Obviously, NMS may be used in physiotherapy as a therapeutic approach to train paretic muscles. If one is particularly interested in functional gain, NMES should always be part of a task-oriented physiotherapy program. In patients with severe motor dysfunction, cyclic NMS can be a means to achieve other therapeutic effects, such as reducing muscle spasticity.

\section{Neuromodulation of shoulder pain}

Pain in the hemiplegic shoulder (hemiplegic shoulder pain [HSP]) is a common phenomenon after stroke. Reported rates of occurrence vary between 5 and $84 \%[83,84]$. Recent studies reported an incidence rate of $25 \%$ in the first 2 weeks after stroke [85] and $22-40 \%$ within 4 months after stroke [86,87]. Persistent HSP interferes with rehabilitation treatment and has been associated with poorer rehabilitation outcomes and prolonged hospital stay in the acute phase of stroke $[84,87]$.

The etiology of HSP is, most likely, multifactorial. Glenohumeral subluxation, spasticity, capsulitis and/or tendinitis have all been postulated [83]. External support, simple analgetics, physical therapy, intra-articular corticosteroid injections and therapeutic NMES are used in the management of HSP. However, evidence for the effectiveness of usual best practice care is scarce. The effectiveness of external support in the treatment of HSP is not supported by the literature [88] and, in a RCT, no significant reduction of HSP as a result of a single intra-articular steroid injection was shown [89]. The evaluation of usual care is complicated by the poor methodology of studies, variation in time since injury (acute vs chronic stroke), the characteristics of the injury (flaccid vs spastic shoulder muscles) and the use of different outcome measures (subluxation vs pain-free range of motion and/or rest-pain) [89-91].

\section{Technology for stimulation of shoulder pain}

Surface NMS for shoulder pain has been used since the mid1980s [92]. Usually, the supraspinatus and posterior deltoid are stimulated as these muscles are important in realigning the glenohumeral joint and counteracting existing shoulder subluxation. The stimulation is applied temporarily between 4 weeks 
and 3 months. Although the main goal of the stimulation would be shoulder pain reduction, several studies also investigated the effect on functional outcome measures [60,93,94]. For instance, Church $e t$ al. studied the effect of surface NMES on functional outcome [94]. They conducted a RCT in 167 patients within 10 days after stroke onset. Surface NMS was compared with placebo and upper limb function was evaluated using the Action Research Arm test. They concluded that a surface NMS program in acute stroke patients was not effective and that it could even worsen hand function in severely disabled patients [94,95].

By the end of the 1990s, a new approach to NMES was introduced. Instead of surface electrodes, percutaneous intramuscular placement of electrodes during stimulation ( $p$-NMES; see Box 1) was promoted. At least four different muscles were implanted (i.e., supraspinatus, middle deltoid, posterior deltoid and trapezius). The stimulation was delivered for 6 weeks, $6 \mathrm{~h}$ per day, after which the electrodes were removed. The expected advantages of the percutaneous system were the pain free stimulation, higher patient compliance and better control of the shoulder subluxation. In an uncontrolled trial with eight patients, significant reductions in shoulder pain, pain-free external rotation and subluxation after percutaneous stimulation were found [96]. The system was evaluated in a few trials but is not commercially available.

\section{Clinical evidence}

Two systematic reviews have been published on the use of NMS in shoulder pain. Price and Pandyan performed a systematic review on the evidence for shoulder pain and included four RCTs published between 1994 and 1999. The conclusions of this review were that there is not sufficient evidence to conclude that shoulder pain could be reduced [97]. There were also no effects on other functional outcomes. The only significant effect that was found was the restoration of subluxation. It must be concluded, however, that their review also included studies evaluating the effect of TENS rather than just NMS.

A second systematic review was conducted by Ada and Foongchomcheay [91]. They included six RCTs published between 1986 and 2000. The main conclusion was a significant effect of NMES as an adjunct to conventional therapy on the prevention and reduction of subluxation and function early after stroke. By using NMS as a preventive means, one may prevent the development of a shoulder-hand syndrome and, thereby, a painful shoulder in chronic stroke [91],

The first RCT that compared p-NMES with slings in 61 chronic stroke patients reported a significant pain reduction for at least 6 months post-treatment [98]. A follow-up study reported longterm effects up to 12 months post-treatment [99]. Additionally, it seems p-NMES can improve quality of life of stroke patients [100] and that it might be cost-effective [101]. Although p-NMES seems more effective compared with surface NMES, the additional value of p-NMES needs further consideration. In particular, it may be relevant to explore the use of p-NMES as a preventive means during acute stroke versus its application to reduce pain in chronic resistant shoulder pain.

\section{Implementation barriers}

Despite the evidence for therapeutic benefit, up to now, surface NMES is not implemented for treatment of shoulder pain. This might be explained by the fact that, first, NMES using surface electrodes can lead to pain and muscle fatigue as a result of the high frequency muscle stimulation that is needed to overcome the skin barrier. Second, the long stimulation protocols (up to $6 \mathrm{~h} /$ day) are difficult to achieve in terms of patient compliance, because activities of daily living are restricted during treatment. Third, careful daily electrode placement is necessary, along with donning and doffing of equipment, which places a large burden on caretakers and/or health professionals.

The percutaneous approach could be a solution for the aforementioned problems but, like surface systems, it has not been introduced widely. One reason is that the company producing the device has quit business. One other scientific problem is that, although we may find some clinical evidence, we still do not know if the same conclusions will be drawn in RCTs to be carried out by independent research groups.

In addition, the mechanism by which we intervene should be made clear. That is, do we indeed restore the shoulder subluxation and hence treat the pain, or is it much more complicated. Addressing this question is very relevant to better understand the effects that are lacking from existing therapeutic approaches.

The first objective is that we have to be able to measure and to distinguish local nociceptive pain from neuropathic pain. A review concluded that no consensus exists on how to measure pain post-stroke [102]. This suggests that there is also no consensus on how to distinguish between neuropathic and local nociceptive pain, which hinders appropriate therapy planning. Therefore, the first question to ask is how to discriminate pain in stroke.

\section{New trends in neuroelectronic interfacing \& clinical deployment}

Given the overall limited evidence for NMES in stroke, as well as the described implementation barriers, several important conclusions can be drawn. First, we need to expand our fundamental knowledge of the effects of different NMES strategies, that is the way we have to interact with the neural system to improve function or modulate pain. Second, we need to collect more clinical data and evidence in individuals that are carefully selected for specific forms of NMES based on such profound (patho)physiological insight. Third, in bringing NMES to practice, the actual demands of patients need to be better considered and integrated in technological developments.

\section{Restitution versus substitution of function}

Many recent research efforts in neurorehabilitation have been directed at understanding and manipulating neural plasticity [71,103,104]. A central focus of this research has been a better understanding of the neural substrates underlying functional improvements after stroke. In addition, brain research has led to some first indications of changes in motor cortex excitability and activation as a result of training [105,106]. A recent review has 
summarized the evidence for changes in the sensorimotor cortex in humans, based on fMRI and transcranial magnetic stimulation (TMS) findings in 13 studies investigating upper limb training [107]. They concluded that neural plasticity can be expected from movement therapy. In particular, they found evidence of this in chronic stroke survivors. However, the neurobiological basis needs to be understood. Studies by different groups demonstrated that use-dependent plasticity is mostly related to the NMDA and GABAergic system [103,108-110].

However, the precise causal relationship between, for example, fMRI changes and actual motor control is still poorly understood. Only recently, some studies were published investigating the motor recovery pattern longitudinally by means of EMG [111]. The main conclusion from these studies was that a great amount of functional recovery cannot be explained by changes in the abnormal timing of paretic lower limb muscles. Hence, it strongly suggests that changes in neural activity of the brain do not directly relate to improved motor control and that substitution of function may be as important for functional recovery as restitution of function.

\section{Evidence for restitution of function using NMES}

The increased knowledge about neural plasticity and brain mapping studies have led to some first indications of changes in the motor cortex due to stimulation in both healthy subjects $[112,113]$ and in patients $[44,47,46]$. Kimberly et al. investigated whether EMG-triggered NMES resulted in differences in brain activation using fMRI. They performed a controlled trial in 16 patients in which EMG-triggered NMES were compared with sham stimulation using the same device. The authors demonstrated a difference in fMRI activation and increased activation in the somatosensory cortex during active finger movements [44].

Barsi $e t$ al. studied the effect of NMES combined with functional exercises on brain activation using TMS [47]. They compared three stimulation paradigms and concluded that voluntary effort combined with NMES has greater potential to induce neural plasticity compared with either voluntary activity alone or stimulation without voluntary activity [47]. The third study comparing EMG-triggered NMES included 14 patients [112] and compared EMG-triggered NMES of the wrist dorsal flexors with a control group not receiving treatment. The authors measured fMRI and calculated the laterality index, and concluded a shift from the ipsi- to the contralateral sensorimotor cortex after 10 weeks of training [46].

\section{Neuropharmacology, motor cortex- \& direct current- stimulation, \& repetitive TMS}

Whereas most of the approaches using NMES do influence brain activity indirectly through movement, it is possible to modulate the motor cortex directly. The most frequently used method to sensitize the motor cortex is through administration of drugs. Mainly two types of drugs have been studied extensively. Martinsson $e t$ al. studied the use of amphetamins to manipulate the noradrenergic system in stroke rehabilitation [114,115]. The other drug class that has been investigated are the selective serotonin-reuptake inhibitors, such as fluoxetine and paroxetine [116]. It has been found that single doses of fluoxetine can modulate both brain activity [117] and motor output [118].

Since early 2000 some studies have been published that investigated modulation of brain activity directly through electrical current $[119,120]$. Roughly, three types of modulation have been described including transcranial direct current (DC) stimulation (tDCS), repetitive TMS (rTMS) and motor cortex stimulation. The underlying thought for using tDCS is that one might be able to increase the excitability of the motor cortex by stimulating it with the anodal cathode, whereas suppression is achieved by using the cathodal electrode. Hummel et al. studied tDCS in a crossover design in six patients. Stimulation was compared with sham stimulation (placebo) and the study concluded that stroke patients' performance on the Jebsen-Taylor test improved during and shortly after stimulation. The other, noninvasive, technique for cortical stimulation is rTMS. Khedr et al. published a controlled trial in 53 acute stroke patients randomized to either rTMS or placebo rTMS [121]. rTMS was given daily for 10 days in addition to regular therapies. The outcomes reported were the Barthel index and Scandinavian stroke scale, and they conclude that patients given rTMS were better off compared with sham rTMS. However, patients were followed for only 10 days after rTMS treatment. Finally, an invasive procedure was proposed using motor cortex stimulation [122]. Guided by fMRI, an epidural electrode was implanted for 6 weeks just above the hand motor cortex area. Phase II studies in ten patients showed that the procedure was safe and well tolerated, and that clinical scales did improve [123].

\section{Expert commentary \& five-year view}

Almost 50 years have passed since the first application of electrical stimulation took place in a neural prosthesis for patients with stroke. Since that time, researchers have been puzzled with questions concerning dose response, stimulation type, carryover effects and implementation problems. Gradually, the focus seems to have shifted from the use of 'true' neural prostheses, 'merely' substituting lost functions, towards NMES as a means to improve muscle function, modulate pain and to - albeit indirectly - improve brain function. Yet, up to now, the clinical evidence obtained from large clinical trials does not support a real breakthrough of NMES in stroke rehabilitation. On the other hand, we can conclude that NMES has at least similar beneficial effects compared with other available rehabilitation strategies and may be preferred by patients. The following two scientific trends can be expected to expand in the next 5 years.

\section{Modulating the neural system}

Given the increased possibilities in functional brain mapping, it is likely that we will gain an increased understanding of the neural substrates of motor relearning and functional recovery, and of the specificity of various rehabilitation approaches. Thus, we will be able to better understand which modulation of brain activity will potentially lead to functional improvements. In particular, a better discrimination between targeting at restitution 
and substitution of function, based on the time post-stroke and on relevant prognostic factors, will allow us to tailor rehabilitation efforts and means to the potential capacities of individual patients. By carefully selecting patients for clinical research based on individual prognostic profiles, we will be able to enhance the effect sizes of several new treatment strategies, such as NMES, that have already shown preliminary efficacy. In addition, if we take more account of the individual needs of patients, the effectiveness of these treatment strategies in daily practice will also substantially improve.

\section{Convergence of therapeutic approaches to enhance neural plasticity}

Given the amount of inconsistency in clinical trials and systematic reviews on NMES, we anticipate the need for more rigorous scientific evidence. NMES may be one means to promote motor relearning, however, it may not be sufficient. Present motor relearning theories support evidence for high intensity, task-specific and goal-oriented training [71]. The next step for electrical stimulation is, therefore, to place it in the context of the available concurrent strategies. It is understandable that many have investigated NMES as a single intervention in order to address the specificity of its mechanism. However, we may very well ask the question whether NMES should be applied as a single option or in combination with other approaches, creating a highly intensive and task-specific training paradigm [74,124]. We expect that this type of combination of interventions will be studied more frequently in the next 5 years.

\section{Clinical \& economic evidence}

Although the mechanisms behind motor relearning and functional recovery should receive ongoing attention, we also need better clinical evidence. In particular, from the point of view of patients, clinicians, insurance companies and health policy makers, more profound evidence is greatly needed as many conclusions of studies are based on belief rather than on scientific reasoning, clinical or economic evidence. Most clinical trials on either NMES or neural prostheses were small and can, therefore, be considered as pilot studies. By definition, these studies tend to report false-positive outcomes. It is recommended to conduct larger trials using larger and more homogeneous samples of patients. By doing so, it will be possible to obtain clinical evidence on particular subgroups of stroke patients who are susceptible to specific intervention strategies. In this regard, we would encourage journal editors to make a clear distinction between Phase I and II studies, aimed at studying the safety, feasibility and potential efficacy of new approaches, and Phase III studies aimed at generating true clinical evidence.

\section{Financial \& competing interests disclosure}

The authors have no direct relation with any of the companies and/or commercial products mentioned in the manuscript, except for discounts in purchase of devices for clinical and research purposes and small amounts offunding (<€30,000) obtained from Otto Bock (Alexander CH Geurts), Neurocontrol Inc. (Maarten J IJzerman and Gerbert J. Renzenbrink) and NESS Ltd (Alexander CH Geurts and Maarten I IJzerman) to carry out independent research on neuroprosthetic and NMES devices. The authors have no other relevant affliations or financial involvement with any organization or entity with a financial interest in or financial conflict with the subject matter or materials discussed in the manuscript apart from those disclosed.

No writing assistance was utilized in the production of this manuscript.

\section{Key issues}

- Neuromuscular electrical stimulation (NMES) in stroke rehabilitation has mainly been used for the possible therapeutic, or carryover, effect. Drop-foot stimulation is, as yet, the only 'widespread' neuroprosthetic application. The appropriate application of neural prostheses requires a well experienced rehabilitation team.

- Stimulation of the peroneal nerve for drop-foot was introduced approximately 50 years ago. Systematic reviews have reported an increased walking speed of between 20-30\%. Both implantable and surface systems are commercially available, but the actual clinical use very much depends on the individual needs and the value of alternatives, such as an ankle-foot orthosis.

- Surface NMES for motor relearning of the upper extremity has been used extensively, but has led to controversial clinical evidence. Even systematic reviews have provided inconsistent conclusions, but most experts accept beneficial effects of NMES on motor control. Systematic reviews on the surplus value of electromyogram-triggered NMS are still inconclusive.

- There is conflicting evidence concerning whether NMES can be used to treat shoulder pain. Both surface and percutaneous stimulation of the shoulder muscles seem to reduce shoulder subluxation. Preliminary clinical data suggest longer and better effects from percutaneous stimulation, but more evidence is required.

- The use of NMES in stroke rehabilitation should move towards modulation of the neural system, including the brain itself and, hence, be used as an adjunct to mainstream rehabilitation approaches.

- The next 5 years of research will provide novel insights into how we can manipulate motor control and functional recovery by neuromodulation of brain tissue. There will be more studies investigating effects of either drugs or electrical current to sensitize the motor cortex to improve the effects of peripheral stimulation and functional training.

- Besides novel insights into specificity and mechanisms of neuromodulation we need more rigorous clinical and economic evidence to convince practitioners and healthcare decision makers to implement technologies using neuromuscular stimulation. 


\section{References}

1 Piccolino M. Luigi Galvani and animal electricity: two centuries after the foundation of electrophysiology. Trends Neurosci. 20(10), 443-448 (1997).

2 Liberson WT, Holmquest HJ, Scot D, Dow M. Functional electrotherapy: stimulation of the peroneal nerve synchronized with the swing phase of the gait of hemiplegic patients. Arch. Phys. Med. Rehabil. 42, 101-105 (1961).

3 Peckham PH, Knutson JS. Functional electrical stimulation for neuromuscular applications. Annu. Rev. Biomed. Eng. 7, 327-360 (2005).

4 Wan BK, Li J, Ming D et al. [Research on the progress of neuroprosthesis for the limb motor system]. Zhongguo Yi Liao Qi Xie Za Zhi 30(4), 235-240 (2006).

5 Popovic MR, Popovic DB, Keller T. Neuroprostheses for grasping. Neurol. Res. 24(5), 443-452 (2002).

6 Sujith OK. Functional electrical stimulation in neurological disorders. Eur. J. Neurol. 15(5), 437-444 (2008).

7 Burridge JH, Ladouceur M. Clinical and therapeutic applications of neuromuscular stimulation: a review of current use and speculation into future developments. Neuromodulation 4, 147-154 (2001).

8 Davis R. Twenty-eight years of clinical experience with implantable neuroprostheses for various applications. Artif. Organs 26(3), 280-283 (2002).

9 Paul L, Rafferty D, Young S et al. The effect of functional electrical stimulation on the physiological cost of gait in people with multiple sclerosis. Mult. Scler. 14(7), 954-961 (2008).

10 Ragnarsson KT. Functional electrical stimulation after spinal cord injury: current use, therapeutic effects and future directions. Spinal Cord 46(4), 255-274 (2008).

11 Dimitrijevic MR. Clinical practice of functional electrical stimulation: from "yesterday" to “today”. Artif. Organs 32(8), 577-580 (2008).

12 Morita I, Keith MW, Kanno T. Reconstruction of upper limb motor function using functional electrical stimulation (FES). Acta Neurochir. Suppl. 97(Pt 1), 403-407 (2007).

13 Daly JJ, Ruff RL. Construction of efficacious gait and upper limb functional interventions based on brain plasticity evidence and model-based measures for stroke patients. ScientificWorldJournal 7, 2031-2045 (2007).
14 Khadilkar A, Phillips K, Jean N et al. Ottawa panel evidence-based clinical practice guidelines for post-stroke rehabilitation. Top. Stroke Rehabil. 13(2), 1-269 (2006).

15 Lindsay P, Bayley M, McDonald A et al. Toward a more effective approach to stroke: Canadian best practice recommendations for stroke care. CMAJ 178(11), 1418-1425 (2008).

16 Bates B, Choi JY, Duncan PW et al. Veterans Affairs/Department of Defense clinical practice guideline for the management of adult stroke rehabilitation care: executive summary. Stroke 36(9), 2049-2056 (2005).

17 Duncan PW, Zorowitz R, Bates B et al. Management of adult stroke rehabilitation care: a clinical practice guideline. Stroke 36(9), e100-e143 (2005).

18 Burridge JH, Wood DE, Taylor PN, McLellan DL. Indices to describe different muscle activation patterns, identified during treadmill walking, in people with spastic drop-foot. Med. Eng. Phys. 23(6), 427-434 (2001).

19 Veltink PH, Slycke P, Hemssems J et al. Three dimensional inertial sensing of foot movements for automatic tuning of a two-channel implantable drop-foot stimulator. Med. Eng. Phys. 25(1), 21-28 (2003).

20 Haugland $M$, Sinkjaer T. Interfacing the body's own sensing receptors into neural prosthesis devices. Technol. Health Care 7(6), 393-399 (1999).

21 Lyons GM, Sinkjaer T, Burridge JH, Wilcox DJ. A review of portable FES-based neural orthoses for the correction of drop foot. IEEE Trans. Neural Syst. Rehabil. Eng. 10(4), 260-279 (2002).

22 Weber DJ, Stein RB, Chan KM et al. BIONic WalkAide for correcting foot drop. IEEE Trans. Neural Syst. Rehabil. Eng. 13(2), 242-246 (2005).

23 Burridge JH, Taylor PN, Hagan SA, Wood DE, Swain ID. The effects of common peroneal stimulation on the effort and speed of walking: a randomized controlled trial with chronic hemiplegic patients. Clin. Rehabil. 11(3), 201-210 (1997).

24 Taylor PN, Burridge JH, Dunkerley AL et al. Clinical use of the Odstock dropped foot stimulator: its effect on the speed and effort of walking. Arch. Phys. Med. Rehabil. 80 (12), 1577-1583 (1999).

25 Taylor PN, Burridge JH, Dunkerley AL et al. Patients' perceptions of the Odstock Dropped Foot Stimulator (ODFS). Clin.

\section{Rehabil. 13(5), 439-446 (1999).}

26 Kottink AI, Oostendorp LJ, Buurke JH et al. The orthotic effect of functional electrical stimulation on the improvement of walking in stroke patients with a dropped foot: a systematic review. Artif. Organs 28(6), 577-586 (2004).

27 Robbins SM, Houghton PE, Woodbury MG, Brown JL. The therapeutic effect of functional and transcutaneous electric stimulation on improving gait speed in stroke patients: a meta-analysis. Arch. Phys. Med. Rehabil. 87(6), 853-859 (2006).

28 Hausdorff JM, Ring H. Effects of a new radio frequency-controlled neuroprosthesis on gait symmetry and rhythmicity in patients with chronic hemiparesis. Am. J. Phys. Med. Rehabil. 87(1), 4-13 (2008).

29 Burridge JH, Haugland M, Larsen B et al. Phase II trial to evaluate the ActiGait implanted drop-foot stimulator in established hemiplegia. J. Rehabil. Med. 39(3), 212-218 (2007).

30 Kottink AI, Hermens HJ, Nene AV et al. Therapeutic effect of an implantable peroneal nerve stimulator in subjects with chronic stroke and footdrop: a randomized controlled trial. Phys. Ther. 88(4), 437-448 (2008).

31 Kottink AI, Hermens HJ, Nene AV et al. A randomized controlled trial of an implantable 2-channel peroneal nerve stimulator on walking speed and activity in poststroke hemiplegia. Arch. Phys. Med. Rehabil. 88(8), 971-978 (2007).

32 Hijmans JM, Geertzen JH. Development of clinical guidelines for the prescription of orthoses in patients with neurological disorders in The Netherlands. Prosthet. Orthot. Int. 30(1), 35-43 (2006).

33 de Wit DC, Buurke JH, Nijlant JM, IJzerman MJ, Hermens HJ. The effect of an ankle-foot orthosis on walking ability in chronic stroke patients: a randomized controlled trial. Clin. Rehabil. 18(5), 550-557 (2004).

34 Sheffler LR, Hennessey MT, Naples GG, Chae J. Peroneal nerve stimulation versus an ankle foot orthosis for correction of footdrop in stroke: impact on functional ambulation. Neurorehabil. Neural Repair 20(3), 355-360 (2006).

35 Fernandes MR, Carvalho LB, Prado GF. A functional electric orthesis on the paretic leg improves quality of life of stroke patients. Arq. Neuropsiquiatr. 64(1), 20-23 (2006).

36 Williams LS, Weinberger M, Harris LE, Clark DO, Biller J. Development of a 
stroke-specific quality of life scale. Stroke 30(7), 1362-1369 (1999).

37 Condie D, Campbell J, Martina J. Report of a consensus conference on the orthotic management of stroke patients. International Society for Prosthetics and Orthotics. Copenhagen, Denmark (2004).

38 van Til JA, Renzenbrink GJ, Dolan JG, Ijzerman MJ. The use of the analytic hierarchy process to aid decision making in acquired equinovarus deformity. Arch. Phys. Med. Rehabil. 89(3), 457-462 (2008).

39 Chae J, Hart R. Intramuscular hand neuroprosthesis for chronic stroke survivors. Neurorehabil. Neural Repair 17(2), 109-117 (2003).

40 Chae J, Sheffler L, Knutson J. Neuromuscular electrical stimulation for motor restoration in hemiplegia. Top. Stroke Rehabil. 15(5), 412-426 (2008).

41 IJzerman MJ, Stoffers TS, Groen FA et al. The NESS Handmaster orthosis: restoration of handfunction in $\mathrm{C} 5$ and stroke patients using electrical stimulation. J. Rehab. Sci. 9(3), 86-89 (1996).

42 Meijer JW, Voerman GE, Santegoets KM, Geurts AC. Short term effects and long term use of a hybrid orthosis for neuromuscular electrical stimulation of the upper extremity in patients after chronic stroke. J. Rehabil. Med. 41(3), 157-161 (2009).

43 Dimitrijevic MM, Stokic DS, Wawro AW, Wun CC. Modification of motor control of wrist extension by mesh-glove electrical afferent stimulation in stroke patients. Arch. Phys. Med. Rehabil. 77(3), 252-258 (1996).

44 Kimberley TJ, Lewis SM, Auerbach EJ et al. Electrical stimulation driving functional improvements and cortical changes in subjects with stroke. Exp. Brain Res. 154(4), 450-460 (2004).

45 Dobkin BH. Do electrically stimulated sensory inputs and movements lead to long-term plasticity and rehabilitation gains? Curr. Opin. Neurol. 16(6), 685-691 (2003).

46 Shin HK, Cho SH, Jeon HS et al. Cortical effect and functional recovery by the electromyography-triggered neuromuscular stimulation in chronic stroke patients. Neurosci. Lett. 442(3), 174-179 (2008).

47 Barsi GI, Popovic DB, Tarkka IM, Sinkjaer T, Grey MJ. Cortical excitability changes following grasping exercise augmented with electrical stimulation. Exp. Brain Res. 191(1), 57-66 (2008).
48 Chae J. Neuromuscular electrical stimulation for motor relearning in hemiparesis. Phys. Med. Rehabil. Clin. N. Am. 14(1 Suppl.), S93-S109 (2003).

49 Chae J, Yu D. A critical review of neuromuscular electrical stimulation for treatment of motor dysfunction in hemiplegia. Assist. Technol. 12(1), 33-49 (2000).

50 de Kroon JR, van der Lee JH, IJzerman MJ, Lankhorst GJ. Therapeutic electrical stimulation to improve motor control and functional abilities of the upper extremity after stroke: a systematic review. Clin. Rehabil. 16(4), 350-360 (2002).

51 Kraft GH, Fitts SS, Hammond MC. Techniques to improve function of the arm and hand in chronic hemiplegia. Arch. Phys. Med. Rehabil. 73(3), 220-227 (1992).

52 de Kroon JR, IJzerman MJ, Chae J, Lankhorst GJ, Zilvold G. Relation between stimulation characteristics and clinical outcome in studies using electrical stimulation to improve motor control of the upper extremity in stroke. J. Rehabil. Med. 37(2), 65-74 (2005).

53 Van Peppen RP, Kwakkel G, WoodDauphinee $S$ et al. The impact of physical therapy on functional outcomes after stroke: what's the evidence? Clin. Rehabil. 18(8), 833-862 (2004).

54 Urton ML, Kohia M, Davis J, Neill MR. Systematic literature review of treatment interventions for upper extremity hemiparesis following stroke. Oсcup. Ther. Int. 14(1), 11-27 (2007).

55 Aoyagi Y, Tsubahara A. Therapeutic orthosis and electrical stimulation for upper extremity hemiplegia after stroke: a review of effectiveness based on evidence. Top. Stroke Rehabil. 11(3), 9-15 (2004).

56 Barreca S, Wolf SL, Fasoli S, Bohannon R. Treatment interventions for the paretic upper limb of stroke survivors: a critical review. Neurorehabil. Neural Repair 17(4), 220-226 (2003).

57 Glanz M, Klawansky S, Stason W, Berkey C, Chalmers TC. Functional electrostimulation in poststroke rehabilitation: a meta-analysis of the randomized controlled trials. Arch. Phys. Med. Rehabil. 77(6), 549-553 (1996).

58 Kimberley TJ, Carey JR. Neuromuscular electrical stimulation in stroke rehabilitation. Minn. Med. 85(4), 34-37 (2002).

59 Sheffler LR, Chae J. Neuromuscular electrical stimulation in neurorehabilitation. Muscle Nerve 35(5), 562-590 (2007).

60 Wang RY. Neuromodulation of effects of upper limb motor function and shoulder range of motion by functional electric stimulation (FES). Acta Neurochir. Suppl. 97(Pt 1), 381-385 (2007).

61 Johansson BB, Haker E, von Arbin M et al. Acupuncture and transcutaneous nerve stimulation in stroke rehabilitation: a randomized, controlled trial. Stroke 32(3), 707-713 (2001).

62 Sonde L, Kalimo H, Fernaeus SE, Viitanen M. Low TENS treatment on post-stroke paretic arm: a three-year follow-up. Clin. Rehabil. 14(1), 14-19 (2000).

63 Tekeoglu Y, Adak B, Goksoy T. Effect of transcutaneous electrical nerve stimulation (TENS) on Barthel Activities of Daily Living (ADL) index score following stroke. Clin. Rehabil. 12(4), 277-280 (1998).

64 Bolton DA, Cauraugh JH, Hausenblas HA. Electromyogram-triggered neuromuscular stimulation and stroke motor recovery of arm/hand functions: a meta-analysis. J. Neurol. Sci. 223(2), 121-127 (2004).

65 Hemmen B, Seelen HA. Effects of movement imagery and electromyographytriggered feedback on arm hand function in stroke patients in the subacute phase. Clin. Rehabil. 21(7), 587-594 (2007).

66 de Kroon JR, IJzerman MJ. Electrical stimulation of the upper extremity in stroke: cyclic versus EMG-triggered stimulation. Clin. Rehabil. 22(8), 690-697 (2008).

67 de Kroon JR, IJzerman MJ, Lankhorst GJ, Zilvold G. Electrical stimulation of the upper limb in stroke: stimulation of the extensors of the hand vs. alternate stimulation of flexors and extensors. Am. J. Phys. Med. Rehabil. 83(8), 592-600 (2004).

68 Meilink A, Hemmen B, Seelen HA, Kwakkel G. Impact of EMG-triggered neuromuscular stimulation of the wrist and finger extensors of the paretic hand after stroke: a systematic review of the literature. Clin. Rehabil. 22(4), 291-305 (2008).

69 Pomeroy VM, King L, Pollock A, Baily-Hallam A, Langhorne P. Electrostimulation for promoting recovery of movement or functional ability after stroke. Cochrane Database Syst. Rev. 2, CD003241 (2006).

70 Kwakkel G, van Peppen R, Wagenaar RC et al. Effects of augmented exercise therapy time after stroke: a meta-analysis. Stroke 
35(11), 2529-2539 (2004).

71 Kwakkel G, Kollen B, Lindeman E. Understanding the pattern of functional recovery after stroke: facts and theories. Restor. Neurol. Neurosci. 22(3-5), 281-299 (2004).

72 Dobkin BH. Strategies for stroke rehabilitation. Lancet Neurol. 3(9), 528-536 (2004).

73 Popovic MB, Popovic DB, Sinkjaer T, Stefanovic A, Schwirtlich L. Restitution of reaching and grasping promoted by functional electrical therapy. Artif. Organs 26(3), 271-275 (2002).

74 Freeman CT, Hughes AM, Burridge JH et al. A robotic workstation for stroke rehabilitation of the upper extremity using FES. Med. Eng. Phys. 31(3), 364-373 (2008).

75 Alon G, Sunnerhagen KS, Geurts AC, Ohry A. A home-based, self-administered stimulation program to improve selected hand functions of chronic stroke. NeuroRehabilitation 18(3), 215-225 (2003).

76 Hendricks HT, IJzerman MJ, de Kroon JR, in 't Groen FA, Zilvold G. Functional electrical stimulation by means of the 'NESS Handmaster Orthosis' in chronic stroke patients: an exploratory study. Clin. Rehabil. 15(2), 217-220 (2001).

77 Cauraugh JH, Kim S. Two coupled motor recovery protocols are better than one: electromyogram-triggered neuromuscular stimulation and bilateral movements. Stroke 33(6), 1589-1594 (2002).

78 Cauraugh JH, Kim SB, Duley A. Coupled bilateral movements and active neuromuscular stimulation: intralimb transfer evidence during bimanual aiming. Neurosci. Lett. 382(1-2), 39-44 (2005).

79 Knutson JS, Harley MY, Hisel TZ, Chae J. Improving hand function in stroke survivors: a pilot study of contralaterally controlled functional electric stimulation in chronic hemiplegia. Arch. Phys. Med. Rehabil. 88(4), 513-520 (2007).

80 Davis R, Sparrow O, Cosendai G et al. Poststroke upper-limb rehabilitation using 5 to 7 inserted microstimulators: implant procedure, safety, and efficacy for restoration of function. Arch. Phys. Med. Rehabil. 89(10), 1907-1912 (2008).

81 Turk R, Burridge JH, Davis R et al. Therapeutic effectiveness of electric stimulation of the upper-limb poststroke using implanted microstimulators. Arch. Phys. Med. Rehabil. 89(10), 1913-1922 (2008).
82 Hendricks HT, van Limbeek J, Geurts AC, Zwarts MJ. Motor recovery after stroke: a systematic review of the literature. Arch. Phys. Med. Rehabil. 83(11), 1629-1637 (2002).

83 Walsh K. Management of shoulder pain in patients with stroke. Postgrad. Med.J. 77(912), 645-649 (2001).

84 Turner-Stokes L, Jackson D. Shoulder pain after stroke: a review of the evidence base to inform the development of an integrated care pathway. Clin. Rehabil. 16(3), 276-298 (2002).

85 Gamble GE, Barberan E, Bowsher D, Tyrrell PJ, Jones AK. Post stroke shoulder pain: more common than previously realized. Eur. J. Pain 4(3), 313-315 (2000).

86 Lindgren I, Jonsson AC, Norrving B, Lindgren A. Shoulder pain after stroke: a prospective population-based study. Stroke 38(2), 343-348 (2007).

87 Gamble GE, Barberan E, Laasch HU et al. Poststroke shoulder pain: a prospective study of the association and risk factors in 152 patients from a consecutive cohort of 205 patients presenting with stroke. Eur. J. Pain 6(6), 467-474 (2002).

88 Ada L, Goddard E, McCully J, Stavrinos T, Bampton J. Thirty minutes of positioning reduces the development of shoulder external rotation contracture after stroke: a randomized controlled trial. Arch. Phys. Med. Rehabil. 86(2), 230-234 (2005).

89 Snels IA, Beckerman H, Twisk JW et al. Effect of triamcinolone acetonide injections on hemiplegic shoulder pain: a randomized clinical trial. Stroke 31(10), 2396-2401 (2000).

90 Price CI, Pandyan AD. Electrical stimulation for preventing and treating post-stroke shoulder pain. Cochrane Database Syst. Rev. 4, CD001698 (2000).

91 Ada L, Foongchomcheay A. Efficacy of electrical stimulation in preventing or reducing subluxation of the shoulder after stroke: a meta-analysis. Aust. J. Physiother. 48(4), 257-267 (2002).

92 Baker LL, Parker K. Neuromuscular electrical stimulation of the muscles surrounding the shoulder. Phys. Ther. 66(12), 1930-1937 (1986).

93 Wang RY, Yang YR, Tsai MW, Wang WT, Chan RC. Effects of functional electric stimulation on upper limb motor function and shoulder range of motion in hemiplegic patients. Am. J. Phys. Med. Rehabil. 81(4), 283-290 (2002).

94 Church C, Price C, Pandyan AD et al. Randomized controlled trial to evaluate the effect of surface neuromuscular electrical stimulation to the shoulder after acute stroke. Stroke 37(12), 2995-3001 (2006).

95 Page SJ, Chae J. Surface electrical stimulation for the affected shoulder after stroke: reconsidering the findings of Church and colleagues. Stroke 38(8), e71; author reply e72-e73 (2007).

96 Yu DT, Chae J, Walker ME, Fang ZP. Percutaneous intramuscular neuromuscular electric stimulation for the treatment of shoulder subluxation and pain in patients with chronic hemiplegia: a pilot study. Arch. Phys. Med. Rehabil. 82(1), 20-25 (2001).

97 Price CI, Pandyan AD. Electrical stimulation for preventing and treating post-stroke shoulder pain: a systematic Cochrane review. Clin. Rehabil. 15(1), 5-19 (2001).

98 Yu DT, Chae J, Walker ME et al. Intramuscular neuromuscular electric stimulation for poststroke shoulder pain: a multicenter randomized clinical trial. Arch. Phys. Med. Rehabil. 85(5), 695-704 (2004).

99 Chae J, Yu DT, Walker ME et al. Intramuscular electrical stimulation for hemiplegic shoulder pain: a 12-month follow-up of a multiple-center, randomized clinical trial. Am. J. Phys. Med. Rehabil. 84(11), 832-842 (2005).

100 Renzenbrink GJ, IJzerman MJ. Percutaneous neuromuscular electrical stimulation (P-NMES) for treating shoulder pain in chronic hemiplegia. Effects on shoulder pain and quality of life. Clin. Rehabil. 18(4), 359-365 (2004).

101 van Til JA, Renzenbrink GJ, Groothuis K, IJzerman MJ. A preliminary economic evaluation of percutaneous neuromuscular electrical stimulation in the treatment of hemiplegic shoulder pain. Disabil. Rehabil. 28(10), 645-651 (2006).

102 Roosink M, Renzenbrink GJ, van Dongen RTM et al. Diagnostic Uncertainties in Post-stroke Pain. IASP Press, Glasgow, Scotland (2008).

103 Boroojerdi B, Ziemann U, Chen R, Butefisch CM, Cohen LG. Mechanisms underlying human motor system plasticity. Muscle Nerve 24(5), 602-613 (2001).

104 Dobkin BH. Neurobiology of rehabilitation. Ann. NY Acad. Sci. 1038, 148-170 (2004).

105 Nudo RJ, Wise BM, SiFuentes F, Milliken GW. Neural substrates for the effects of rehabilitative training on motor recovery after ischemic infarct. Science 272(5269), 


\section{1-1794 (1996).}

106 Nudo RJ. Mechanisms for recovery of motor function following cortical damage. Curr. Opin. Neurobiol. 16(6), 638-644 (2006).

107 Richards LG, Stewart KC, Woodbury ML, Senesac C, Cauraugh JH. Movementdependent stroke recovery: a systematic review and meta-analysis of TMS and fMRI evidence. Neuropsychologia 46(1), 3-11 (2008).

108 Butefisch CM, Davis CJ, Wise SP et al. Mechanisms of use-dependent plasticity in the human motor cortex. Proc. Natl Acad. Sci. USA 97(7), 3661-3665 (2000).

109 Butefisch CM. Neurobiological bases of rehabilitation. Neurol Sci, 27(Suppl. 1), S18-S23 (2006)

110 Ziemann U, Muellbacher W, Hallett M, Cohen LG. Modulation of practicedependent plasticity in human motor cortex. Brain 124(Pt 6), 1171-1181 (2001).

111 Buurke JH, Nene AV, Kwakkel G et al. Recovery of gait after stroke: what changes? Neurorehabil. Neural Repair 22, 676-683 (2008).

112 Blickenstorfer A, Kleiser R, Keller T et al. Cortical and subcortical correlates of functional electrical stimulation of wrist extensor and flexor muscles revealed by fMRI. Hum. Brain Mapp. (2008).

113 Han BS, Jang SH, Chang Y et al. Functional magnetic resonance image finding of cortical activation by neuromuscular electrical stimulation on wrist extensor muscles. Am. J. Phys. Med. Rehabil. 82(1), 17-20 (2003).

114 Martinsson L, Hardemark HG, Wahlgren NG. Amphetamines for improving stroke recovery: a systematic cochrane review. Stroke 34(11), 2766 (2003).

115 Treig T, Werner C, Sachse M, Hesse S. No benefit from D-amphetamine when added to physiotherapy after stroke: a randomized, placebo-controlled study. Clin. Rehabil. 17(6), 590-599 (2003).

116 Mostert JP, Koch MW, Heerings M, Heersema DJ, De Keyser J. Therapeutic potential of fluoxetine in neurological disorders. CNS Neurosci. Ther. 14(2), 153-164 (2008).

117 Pariente J, Loubinoux I, Carel C et al.
Fluoxetine modulates motor performance and cerebral activation of patients recovering from stroke. Ann. Neurol. 50(6), 718-729 (2001)

118 van Genderen HI, Nijlant JM, van Putten MJ, Movig KL, IJzerman MJ. Single dose of fluoxetine increases muscle activation in chronic stroke patients. Clin.

Neuropharmacol. DOI: 10.1097/

WNF.0B013E3181633482 (2008) (Epub ahead of print).

119 Fregni F, Pascual-Leone A. Technology insight: noninvasive brain stimulation in neurology-perspectives on the therapeutic potential of rTMS and tDCS. Nat. Clin. Pract. Neurol. 3(7), 383-393 (2007).

120 Webster BR, Celnik PA, Cohen LG. Noninvasive brain stimulation in stroke rehabilitation. NeuroRx 3(4), 474-481 (2006).

121 Khedr EM, Ahmed MA, Fathy N, Rothwell JC. Therapeutic trial of repetitive transcranial magnetic stimulation after acute ischemic stroke. Neurology 65(3), 466-468 (2005).

122 Harvey RL, Nudo RJ. Cortical brain stimulation: a potential therapeutic agent for upper limb motor recovery following stroke. Top. Stroke Rehabil. 14(6), 54-67 (2007).

123 Brown JA, Lutsep HL, Weinand M, Cramer SC. Motor cortex stimulation for the enhancement of recovery from stroke: a prospective, multicenter safety study. Neurosurgery 62(Suppl. 2), 853-862 (2008).

124 Hesse S, Werner C, Schonhardt EM et al. Combined transcranial direct current stimulation and robot-assisted arm training in subacute stroke patients: a pilot study. Restor. Neurol. Neurosci. 25(1), 9-15 (2007).

\section{Websites}

201 Teassel R. Evidence Based Review of Stroke Rehabilitation. Chapter 11. Painful Hemiplegic Shoulder www.ebrsr.com (Accessed 15 December 2008)

202 Walkaide by Innovative Neurotronics, a Hanger Orthopaedic Group www.walkaide.com
(Accessed January 2009)

203 BIONESS Ltd. Company www.nessltd.com (Accessed January 2009)

204 Otto Bock: neurostimulation ACTIGAIT www.ottobock.com/cps/rde/xchg/ob_ com_en/hs.xsl/1457.html (Accessed January 2009)

205 Finetech Medical Implants www.finetech-medical.co.uk (Accessed January 2009)

206 Functional electrical stimulation for drop-foot of central neurological origin www.nice.org.uk/guidance/index. jsp? action $=$ byID \&o $=11932$ (Accessed 29 October 2008)

207 BION manufactured by Advanced Bionics Inc. Valencia, CA, USA www.advancedbionics.com (Accessed January 2009)

\section{Affiliations}

- Maarten J IJzerman, PhD

University of Twente, School for Management \& Governance, Department of Health Technology \& Services Research, PO Box 217, 7500 AE Enschede,

The Netherlands

Tel.: +31 534893684

m.j.ijzerman@utwente.nl

- Gerbert J Renzenbrink, MD

Roessingh Rehabilitation Center, Stroke Unit, PO Box 310, 7500 AH Enschede,

The Netherlands

Tel.: +31 534875451

b.renzenbrink@roessingh.nl

- Alexander CH Geurts, MD, PhD Radboud University Medical Center, Department for Physical Medicine \& Rehabilitation, PO Box 9011, 6500 GM Nijmegen, The Netherlands Tel.: +31 243614804 a.geurts@reval.umcn.nl 